Arthroskopie 2009 $\cdot 22: 273-278$

DOI 10.1007/s00142-009-0518-y

Online publiziert: 17. September 2009

(c) Springer Medizin Verlag 2009

\section{Redaktion}

M. Dienst, München

\author{
M. Wettstein \\ Service d'Orthopédie et de Traumatologie, Département de l'Appareil \\ Locomoteur, Centre Hospitalier Universitaire Vaudois, Lausanne
}

\title{
Arthroskopie bei Hüftdysplasie
}

Die Hüftdysplasie ist das Resultat einer Reifungsstörung, bei der das Pfannendach $\mathrm{zu}$ steil und oft zu kurz ist, was zu einer ungenügenden Überdachung des Femurkopfs führt. Dem klassischen biomechanischen Modell der Arthroseentstehung entsprechend, subluxiert der Femurkopf nach laterokranial und überbeansprucht den Pfannenrand [36]. Die kausale Behandlung besteht in einer Pfannenreorientierungsosteotomie, mit dem Ziel, die Kontaktfläche zwischen Kopf und Pfanne zu vergrößern [23]. Zur Zeit sind die Dreifachosteotomie nach Tönnis et al. [34] sowie die periazetabuläre Osteotomie nach Ganz et al. [13] die gängigsten Verfahren. Die Resultate sind bei korrekter Planung und Durchführung der anspruchsvollen Operation gut, hängen jedoch wesentlich vom präoperativen Knorpelzustand ab [30, 32]. Manche Patienten können trotz eines gut erhaltenen Knorpels weiter Beschwerden haben. Es ist zu vermuten, dass diese auf intraartikuläre Läsionen zurückzuführen sind, die durch die Osteotomie nicht behoben wurden [1]. Diagnostisch und therapeutisch wird die Hüftarthroskopie auch hier eine zunehmende Rolle spielen.

\section{Pathogenese}

Wegen der steilen Orientierung der Pfanne bei einer Hüftdysplasie subluxiert der Femurkopf progressiv nach laterokranial, was zu einer Überbeanspruchung des Pfannenrandes führt (• Abb. 1; [36]). Hierbei wird nicht nur der Knorpel, sondern auch das Labrum acetabulare überlastet. Dieses wird zuerst hypertroph, degeneriert und reißt schließlich, meistens longitudinal, mit Knorpelschäden am sublab- ralen Pfannenrand. Da das Labrum innerviert ist, sind diese Läsionen schmerzhaft, was von Klaue et al. [18] als „Pfannenrandsyndrom" bezeichnet wurde [17]. Tschauner u. Hofmann [35] prägten das Konzept des Labrums als „Leitplanke“ der Hüfte, da das Labrum bei der Subluxation des Femurkopfs als Zeichen der Überlastung zu den ersten Beschwerden führt. Das morphologische Stadium der Labrumläsion weist auf das biomechanische Entwicklungsstadium der Dysplasie hin [8]:

- im ersten Stadium ist das Labrum als Versuch eines Kompensationsmechanismus verdickt;

- im zweiten Stadium beginnt das Labrum als Zeichen der beginnenden Dekompensation an der gelenkseitigen Basis zu reißen;

- im dritten Stadium reißt das Labrum komplett vom Pfannenrand ab, was $\mathrm{zu}$ einem Funktionsverlust führt und das Labrum nur noch als mechanisch störenden Fremdkörper erscheinen lässt [22].

Gleichzeitig kommt es zu progredienten Knorpelschäden und, in Kombination mit der Labrum-Knorpel-Separation, zur Ausbildung intraossärer und paralabraler Zysten.

Die Subluxation des Femurkopfs verursacht auch eine progressive Dehnung des Lig. capitis femoris (LCF), was zu einer Hypertrophie, Degeneration und schließlich Ruptur führt [14].Erst vor kurzem wurde auf ein gleichzeitig mögliches femoroazetabuläres Impingement hingewiesen, das durch eine Retroversion der Pfanne oder einen asphärischen Femurkopf bedingt sein kann $[4,15,21]$.
Die kausale Therapie, eine periazetabuläre Reorientierungsosteotomie, erlaubt die Biomechanik des Gelenks zu korrigieren und somit das weitere Fortschreiten der intraartikulären Schäden zumindest zu bremsen. Die Osteotomie erlaubt auch, die Version der Pfanne und somit ein bevorstehendes Impingement zu korrigieren oder zu vermeiden [29]. Die intraartikulären Schäden sowie eine Asphärizität des Femurkopfs können jedoch über den zur Osteotomie benützten vorderen $\mathrm{Zu}$ gang nur bedingt angegangen werden [3]. Dies erklärt, warum das Resultat manch-

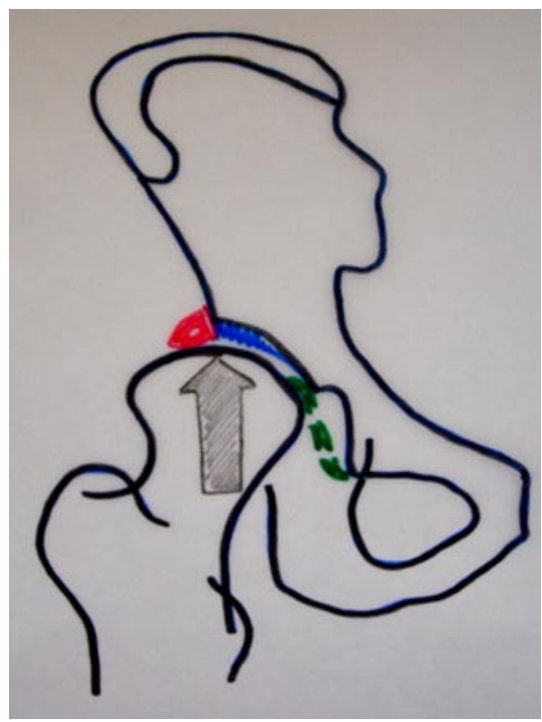

Abb. $1 \Delta$ Biomechanisches Konzept der intraartikulären Läsionen bei Hüftdysplasie. Die chronische Überlastung des Pfannenrandes (vertikaler Pfeil) führt zu einer Labrumhypertrophie und einem -riss (rot) mit gleichzeitiger Knorpelschädigung (blau). Der durch die Subluxation des Femurkopfs verursachte Zug auf das Lig. capitis femoris führt zur Degeneration und später zur Ruptur (grün) 


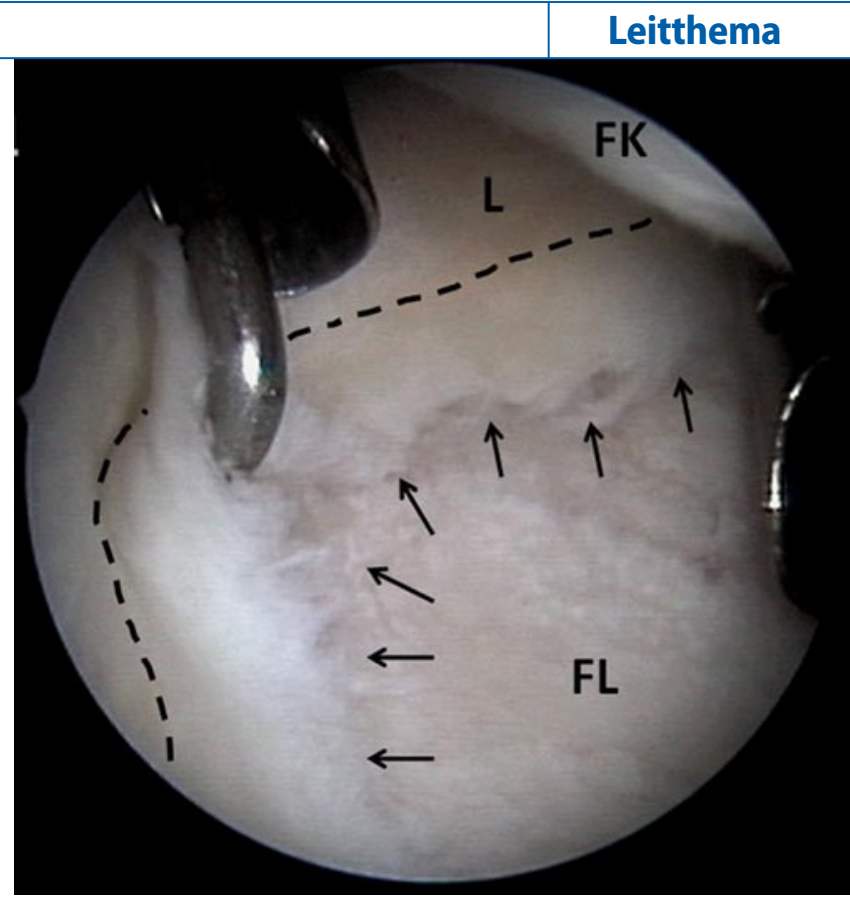

Abb. $2<$ Typische Labrumläsion bei einer 50-jährigen Patientin mit einer Hüftdysplasie. Das Labrum $(L)$ ist hypertroph, vom Pfannenrand (gestrichelte Linie) abgerissen und hängt über den Knorpel des Pfannenrandes (Pfeile) in den Gelenkspalt. Tasthaken über das ventrolaterale Portal vorgeschoben, rechts davon das anteriore Portal. FL Facies lunata, FK Femurkopf

mal trotz eines guten Knorpelstatus nicht befriedigend ausfällt.

\section{Klinische und radiologische Untersuchungen}

Die klinische Untersuchung erfolgt wie bei jeder anderen Hüftpathologie. Charakteristisch für die Dysplasie ist ein großer Bewegungsumfang. Das relative Ausmaß der Außen- und Innenrotation gibt Auskunft über die Schenkelhalsantetorsion: Bei vermehrter Antetorsion ist die Innenrotation vergrößert. Die Labrumprovokationstests, der Impingement- und Apprehensiontest sind bei einem Pfannenrandsyndrom positiv und weisen auf eine symptomatische Labrumläsion oder einen sublabralen Knorpelschaden hin [20]. Diese Tests sind sehr sensitiv, jedoch wenig spezifisch: Andere intraartikuläre Läsionen können auch zu positiven Tests führen, ohne dass deren Ursache genau bestimmt werden kann. Beschwerden sowohl bei Außen- als auch Innenrotation können auf eine Pathologie des LCF weisen, auch diese Tests sind jedoch unspezifisch und schwer zu interpretieren.

Die Beckenübersichts-, eine Fauxprofil- sowie eine Funktionsaufnahme in Korrekturstellung bilden das mindest notwendige an Röntgenbildern. Es muss unbedingt auf eine technisch einwandfreie Realisation dieser Bilder geachtet werden, sodass die Pfannenorientierung, die Gelenkkongruenz, der Arthrosegrad und weitere Veränderungen korrekt beurteilt werden können. Zusätzlich sollten die Winkelwerte gemessen werden, die erlauben, den Schweregrad der Dysplasie zu beurteilen [33]:

- lateraler Zentrum-Erker- (LCE-)Winkel nach Wiberg,

- Tragflächenwinkel (TF) nach Tschauner und

- anteriorer Zentrum-Erker- (ACE-)

Winkel nach Lequesne.

Mithilfe der Magnetresonanztomographie (MRT) oder besser Magnetresonanzarthrographie können die Kollateralschäden an Knorpel, Labrum, Knochen und LCF und somit die Möglichkeit einer gelenkerhaltenden Operation beurteilt werden. Die Labrumschäden werden nach der Klassifikation von Czerny und ihrer Lokalisation, meist lateral und ventrolateral, beschrieben. Die Sensitivität der Magnetresonanzarthrographie beträgt dabei 90\% und die Sensitivität 91\% [24]. Die Darstellung des Knorpels gelingt zunehmend besser und erlaubt die Schäden präoperativ abzuschätzen, was zur Therapieplanung und Aufklärung des Patienten hilfreich ist. Als indirekter Hinweis auf höhergradige Knorpel- und Labrumschäden gilt die Darstellung intraund extraossärer Zysten. Neueste technische Entwicklungen, z. B. die dGEMRIC („delayed gadolinium-enhanced magnetic resonance imaging of cartilage“), erlauben eine Beurteilung der Knorpel- qualität durch die Messung des Glykosaminoglykangehalts [16]. Es konnte gezeigt werden, dass es sich hier um den signifikantesten prognostischen Faktor für den Erfolg oder Misserfolg einer reorientierenden Beckenosteotomie handelt [7]. Teilrupturen des LCF lassen sich oft nur schlecht beurteilen, während degenerative Läsionen oder komplette Rupturen häufig besser dargestellt werden können. Byrd u. Jones [2] konnten in einer retrospektiven Studie zeigen, dass nur 10\% der arthroskopisch identifizierten LCF-Schäden MRtomographisch diagnostiziert worden waren. Trotz einer Verbesserung der Bildgebungsqualität in den letzten Jahren scheinen diese Zahlen immer noch relevant zu sein.

\section{Therapie}

Die kausale Behandlung der Pfannendysplasie besteht in einer Pfannenreorientierungsosteotomie. Das Ziel der Osteotomie ist die Verbesserung der Pfannenorientierung und der Überdachung des Femurkopfs zur Normalisierung der Belastungskräfte. Die üblichen Verfahren sind z. Z. die Berner periazetabuläre Osteotomie und die dreifache Beckenosteotomie nach Tönnis [13, 34]. Beide Verfahren ermöglichen eine dreidimensionale Korrektur mit guten Ergebnissen bei nichtarthrotischen Gelenken [28, 32]. Oft bleiben bei einigen Patienten jedoch Beschwerden bestehen, die auf intraartikuläre Schäden wie Labrum-, Knorpel- oder LCF-Läsionen zurückzuführen sind und während der Osteotomie nur bedingt (Labrum) oder nicht (LCF, Knorpel) behandelt werden können. Bei prä- und postoperativem Verdacht auf eine instabile Labrumruptur, einen Riss des LCF oder unklare Knorpelverhältnisse stellt sich die Indikation zur Hüftarthroskopie. Die radiologischen Untersuchungen sind oft zu ungenau, um das Ausmaß und die exakte Lage der intraartikulären Schäden zu beurteilen. Die Hüftarthroskopie ermöglicht hingegen eine direkte Beurteilung und eventuelle Behandlung.

\section{Diagnostische Hüftarthroskopie}

Vor einer Korrekturosteotomie erleichtert die lateral und häufig auch ventral ver- 
mehrte Öffnung der Pfanne das Eingehen in das zentrale Kompartiment. Es sollte jedoch auf das hypertrophierte Labrum geachtet werden, das vom Pfannenrand in den Gelenkspalt hinein ragt und somit den Spalt teilweise bis komplett versperren kann. Das Risiko einer iatrogenen Labrumperforation ist somit in diesen Fällen erheblich erhöht. Die Technik des Zugangs zum zentralen Kompartiment über die Peripherie scheint hier eine elegante Lösung dieses Problems [9].

Das Labrum muss in seiner Breite und Dicke, die normalerweise ungefähr $5 \mathrm{~mm}$ beträgt, beurteilt werden [27]. Die Hypertrophie ist eine Folge der chronischen Überlastung und das Vorstadium einer Degeneration, die sich als gelbliche Verfärbung und Verformung ausdrückt. Danach kommt es oft zu einem Longitudinalriss, zuerst auf der Gelenkseite mit einem Einriss der Labrum-Knorpel-Begrenzung (Stadium Czerny 2b) und ohne wesentliche Instabilität und zuletzt zu einem kompletten Korbhenkelriss (Stadium Czerny 3 b). Der Riss liegt typisch am ventrolateralen Pfannenrand, zwischen 9 bis 14 Uhr (• Abb. 2). Wegen der chronischen Reizung des Gelenks findet sich oft eine erhebliche Synovitis im perilabralen Sulkus.

Knorpelschäden sind in derselben Region wie Labrumläsionen zu suchen. Im Frühstadium findet man oft eine Knorpelerweichung, die sich bis zu einem vollschichtigen Knorpelschaden entwickeln kann. Entscheidend ist dabei, im Hinblick auf eine geplante Korrekturosteotomie die Ausdehnung des Schadens unter Berücksichtigung der Fehlstellung zu beurteilen. Es muss abgeschätzt werden, ob noch genügend Knorpel guter Qualität in die Belastungszone geschwenkt werden kann oder nicht (• Abb. 3). Bei einem höhergradigen Knorpelschaden von mehr als $1 / 3$ der Breite der Facies lunata scheint es nicht mehr sinnvoll, eine Osteotomie durchzuführen. Knorpelschäden am Femurkopf erscheinen erst später und stellen daher eine Kontraindikation für ein gelenkerhaltendes Verfahren dar, außer die Kombination einer periazetabulären und intertrochantären Osteotomie erlaubt es, den Knorpeldefekt aus dem Belastungsbereich wegzuschwenken.
Wegen der progressiven Subluxation des Femurkopfs sind LCF-Veränderungen bei der Dysplasie häufig. Es muss darauf geachtet werden, ob das Band nur teilweise oder komplett rupturiert ist. Zur Beurteilung muss die Hüfte innen- und außenzurotiert werden. Eine vollständige Avulsion des Femurkopfs ist meistens direkt sichtbar, bei einer kompletten azetabulären Ruptur ist auf eine freie $\mathrm{Be}$ wegung des LCF an seiner Insertion unter dem Lig. transversum zu achten. Inkomplette Rupturen müssen bei Ligamentverdickungen vermutet werden, $\mathrm{zu}$ dem spannen sich die rupturierten Anteile bei Außenrotation nicht adäquat an (• Abb.4).

\section{Therapeutische Hüftarthroskopie}

Verschiedene Studien zeigten, dass das Labrum als Dichtungsring für das Hüftgelenk dient, die Gelenkfläche vergrößert und die Stabilität des Gelenks erhöht [6, $11,12]$. Das Labrum ist besonders in seinen lateralen und ventralen Anteilen nozizeptiv und propriozeptiv innerviert [17]. Die klinische Erfahrung zeigt auch, dass der Erhalt des Labrums zu besseren Resultaten führt als die Labrumresektion [10]. Zudem hat das Labrum bei der Dysplasie eine besondere Funktion als Stabilisator oder Leitplanke der Hüfte [35]. Aus diesen Gründen sollte es so weit als möglich erhalten und gerissene Anteile so sparsam wie möglich bis auf einen stabilen Rand reseziert werden ( $\bullet$ Abb. 5). Bei einem Korbhenkelriss ist es heute technisch möglich, das Labrum an den Pfannenrand zu nähen. Eine solche Refixation macht jedoch nur dann Sinn, wenn die chronische Überlastung des Pfannenrandes durch eine Reorientierungsosteotomie reduziert wird und die Gewebequalität des Labrums genügend ist [25]. Falls keine Osteotomie geplant oder möglich ist, sollte das Labrum so sparsam wie möglich débridiert werden.

Das LCF spielt beim Erwachsenen bei der Blutversorgung des Femurkopfs keine Rolle, trägt jedoch durch seine Innervation zur Stabilisierung des Gelenks bei [19, 26]. Bei Partialrupturen sollte es deshalb so sparsam wie möglich reseziert werden. Bei Komplettrupturen wird hingegen das ganze Band reseziert, um ein schmerz-
Arthroskopie 2009 · 22:273-278

DOI 10.1007/s00142-009-0518-y

○) Springer Medizin Verlag 2009

\section{Wettstein}

Arthroskopie bei Hüftdysplasie

\section{Zusammenfassung}

Die kausale Behandlung der Hüftdysplasie besteht in einer Pfannenreorientierungsosteotomie, deren Resultate stark von den intraartikulären Schäden abhängen. Entscheidender prognostischer Faktor ist der Knorpelstatus, jedoch können Rupturen des Labrum acetabulare und des Lig. capitis femoris auch für Symptome verantwortlich sein. In diesem Zusammenhang bietet die Hüftarthroskopie ein wertvolles Instrument zur präzisen Diagnose und Behandlung dieser Läsionen. Bislang gibt es jedoch noch keine Resultate, die ein solches Vorgehen unterstützen.

\section{Schlüsselwörter}

Hüfte · Arthroskopie · Dysplasie · Kongenital · Behandlung

\section{Arthroscopy in hip dysplasia}

\section{Abstract}

The causal treatment of a residual hip dysplasia consists of an acetabular reorientation osteotomy, the results of which, however, strongly depend on concomitant intra-articular lesions. The most important prognostic factor is the cartilage status. However, tears in the labrum and the ligament of the head of the femur may also be responsible for symptoms. Hip arthroscopy is a valuable tool for a precise diagnosis and simultaneous treatment of these lesions. However, as yet no results supporting this treatment algorithm have been published.

Keywords Hip · Arthroscopy · Dysplasia · Congenital . Treatment 


\section{Leitthema}

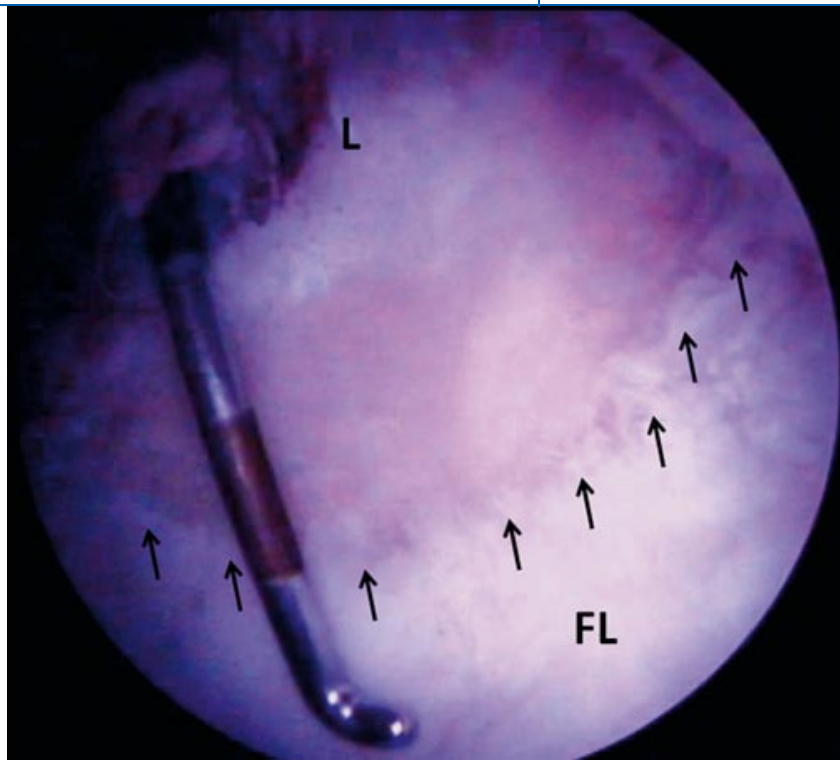

Abb. $3 \Delta$ Typische Knorpelläsion der anterosuperioren Facies lunata $(F L)$ bei einer 40-jährigen Patientin mit Dysplasie. Der Knorpel ist lateral der Abgrenzung durch die Pfeile bis auf den subchondralen Knochen abgeschert. Das Labrum $(L)$ ist nach der Resektion der gerissenen Anteile nur noch als Residuum am Pfannenrand sichtbar. Tasthaken via anteriores Portal platziert

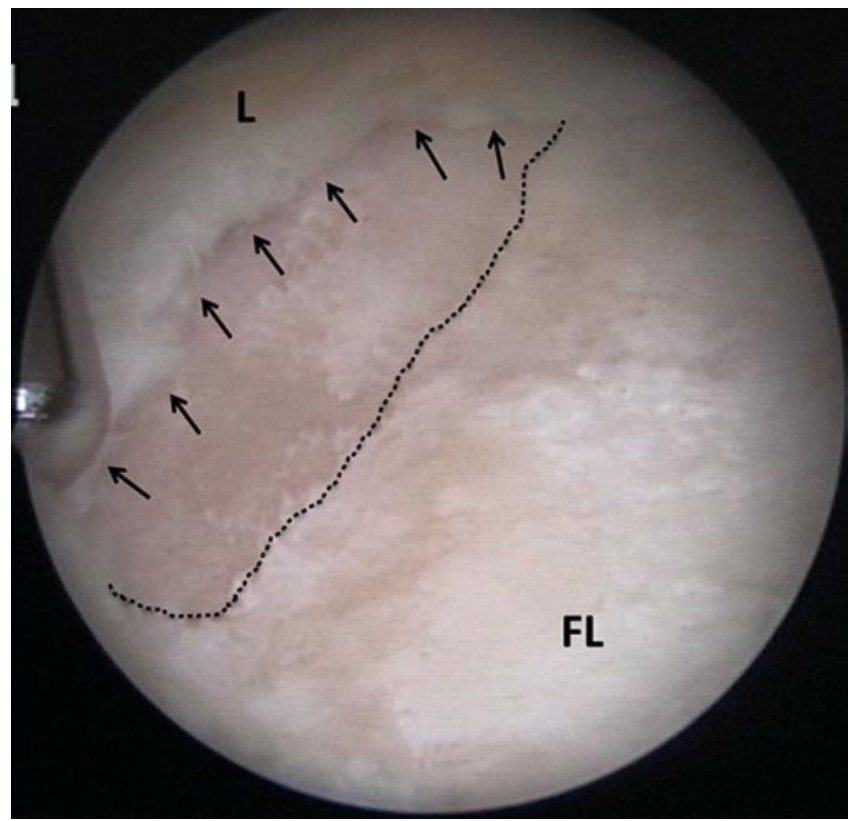

Abb. $5 \Delta$ Zustand nach Labrumteilresektion bei der Patientin aus - Abb. 2. Das Restlabrum ist am Pfannenrand fixiert. Unter dem gerissenen Labrum wurde eine viertgradige Knorpelläsion des Pfannenrandes gefunden. $L$ Labrum, $F L$ Facies lunata

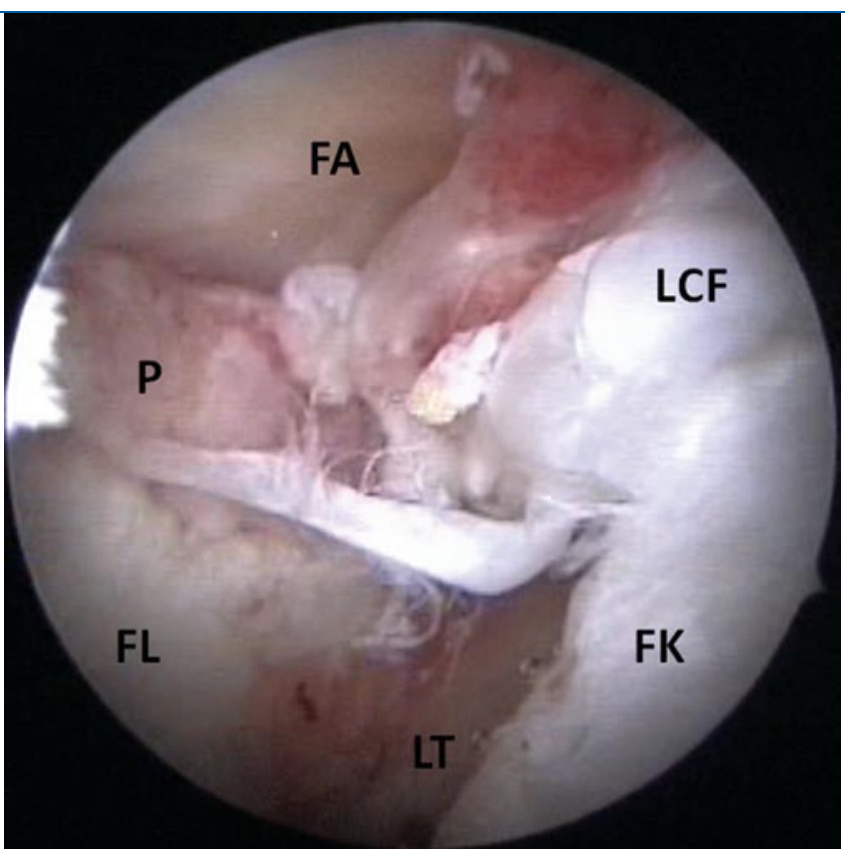

Abb. $4 \Delta$ Teilruptur des Lig. capitis femoris (LCF) an seiner Basis unter dem Lig. transversum (LT). FA Fossa acetabuli, P Pulvinar, FL Facies lunata, FK Femurkopf

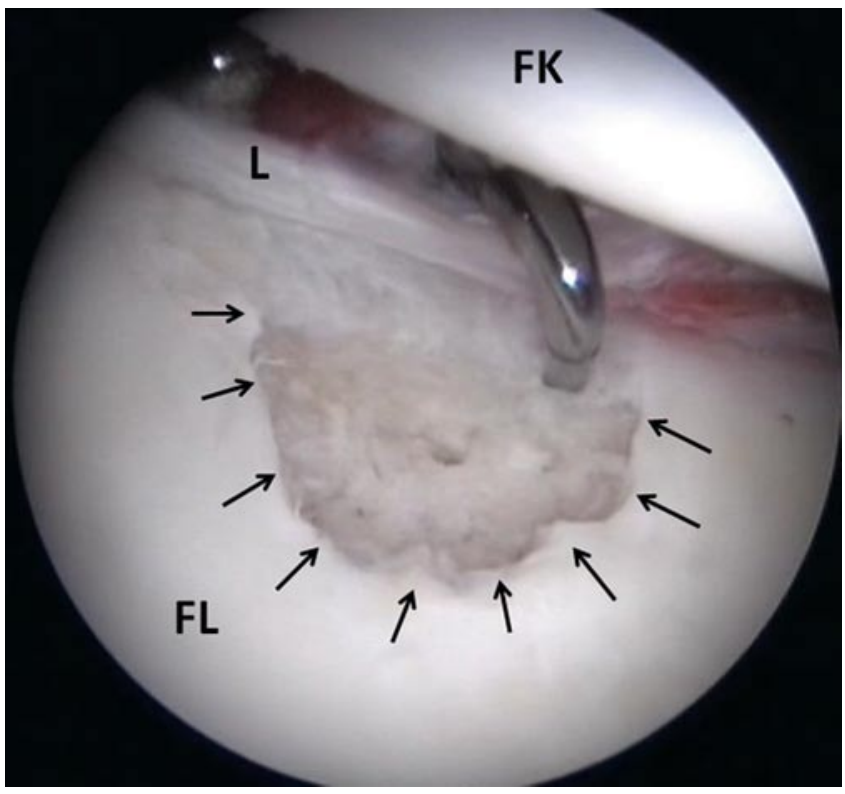

Abb. $6 \Delta$ Viertgradige Knorpelläsion der Facies lunata $(F L)$, durch Pfeile begrenzt, nach Mikrofrakturierung. L Labrum, FK Femurkopf. Die Ahle wurde über das anteriore Portal einegführt, links davon das anterolaterale Portal haftes Einklemmen zwischen Femurkopf und Fossa acetabuli zu verhindern.

Aufweichungen des Knorpel-LabrumÜbergangs erfordern keine Behandlung. Instabile Knorpellappen sollten geglättet werden, um eine mechanische Irritation und ein Einklemmen zu verhindern. Bei weiter fortgeschrittenen Schäden wird der geschädigte Knorpel bis auf einen stabilen Rand débridiert, die subchondrale Lamelle abradiert und mikrofrakturiert (• Abb. 6). Dadurch kann im Defekt eine Faserknorpelnarbe gebildet werden, was jedoch nur bei stabilen mechanischen Verhältnissen erfolgreich ist [31]. Dazu braucht es eine Entlastung des ope- rierten Beins während 6 Wochen, eine passive Bewegungstherapie mit einer motorisierten Schiene und eine frühzeitige Reorientierungsosteotomie, um eine weitere Überlastung des Pfannenrandes zu vermeiden.

Ein femoroazetabuläres Impingement bei Hüftdysplasie wegen einer ventralen 
Offsetverminderung des Schenkelhalses wurde erst kürzlich beschrieben [4, 15]. Bei der Hüftarthroskopie kann das Impingement bestätigt und der Femurkopf rekonturiert werden, womit bei der nachfolgenden Osteotomie eine Arthrotomie nicht mehr nötig ist. Auf eine ventrale Rekonturierung des Azetabulums sollte hingegen vor einer Umstellungsosteotomie verzichtet werden, da die ventrale Überdachung mit der Osteotomie korrigiert werden kann.

Nach einer Korrekturosteotomie ohne vorherige Arthroskopie können die intraartikulären Läsionen weiter bestehen und Beschwerden verursachen. Diese Fälle gehören zu den schlechten Resultaten der Osteotomien. Bei klinischen und radiologischen Zeichen, die auf einen entsprechenden intraartikulären Schaden weisen, bietet die Hüftarthroskopie eine gute Option, zumindest Beschwerden durch irritierende Anteile des Lig. capitis femoris oder Labrum acetabulare zu beheben.

Trotz optimaler Korrekturergebnisse und langzeitiger Beschwerdefreiheit kann die Gelenkdegeneration mit der Zeit weiter voranschreiten. In dieser Situation stellt sich die Frage, ob eine weitere Gelenkerhaltung möglich oder ein endoprothetischer Gelenkersatz nötig ist. Trotz immer besser werdender radiologischer Diagnostik ist die Beurteilung der Knorpelläsionen häufig problematisch. Mit der Arthroskopie kann in diesen Fällen der Knorpel genau beurteilt und entschieden werden, ob ein reines Débridement, eine neue Osteotomie oder eine kurzfristige endoprothetische Versorgung empfohlen werden sollte.

\section{Ergebnisse}

Die Resultate der Korrekturosteotomien hängen wesentlich vom präoperativen Knorpelzustand ab. Nach mehr als 10 Jahren lag die Überlebensrate mit einem Arthrosegrad von o-1 bei $88 \%$. Bei weiter fortgeschrittenen Schäden betrug die Überlebensrate nach 10 Jahren $73 \%$, nach 20 Jahren jedoch nur noch $13 \%$ [32]. Yasunaga et al. [37] zeigten, dass das Resultat einer Reorientierungsosteotomie weniger von der radiologischen Gelenkkongruenz abhängt als von den arthroskopisch diagnostizierten Knorpelläsionen. Die Ar- throskopie ist somit diagnostisch der Radiologie klar überlegen.

Die arthroskopischen Studien bei Hüftdysplasie beschrieben bisher hauptsächlich Labrumläsionen und deren Behandlung. Es wurde nachgewiesen, dass die Resektion des Labrums die Schmerzen signifikant verbesserte [5]. Es wurde jedoch nie darüber berichtet, wie lange die Besserung anhielt und ob eine Reorientierung vorgenommen wurde. In einer Studie mit 48 Patienten fanden Byrd u. Jones [1] keine Unterschiede der Resultate nach 2 Jahren, nach Pathologien geordnet, zwischen grenzwertigen und echten Dysplasien. Die Unterschiede zeigten sich eher in den intraartikulären Pathologien: Bei einer traumatischen Ursache, nach plötzlichem Auftreten, bei jüngeren Patienten, bei LCF-Rissen oder freien Gelenkkörpern waren die Resultate besser als bei Labrum- und Knorpelschäden.

\section{Fazit für die Praxis}

Die Hüftdysplasie wird kausal mittels einer Reorientierungsosteotomie behandelt. Mit der Arthroskopie können ein exakter Knorpelstatus, der der wichtigste prognostische Faktor ist, erhoben sowie mögliche Kollateralschäden des Lig. capitis femoris, des Labrum acetabulare und Offsetstörungen des Kopf-HalsÜbergangs diagnostiziert und behandelt werden. Die Hüftarthroskopie sollte bei einem klinischen oder radiologischen Verdacht auf intraartikuläre Schäden vor einer Osteotomie vorgenommen werden. Das Verfahren ist zu diesem Zeitpunkt technisch einfacher als nach der Osteotomie, zudem kann sie in Grenzsituationen weitere Informationen für oder gegen eine sekundäre Umstellungsosteotomie liefern.

\section{Korrespondenzadresse \\ Dr. M. Wettstein}

Service d'Orthopédie et de Traumatologie,

Département de l'Appareil Locomoteur,

Centre Hospitalier Universitaire Vaudois

CH-1011 Lausanne

Schweiz

Michael.Wettstein@chuv.ch

Interessenkonflikt. Keine Angabe.

\section{Literatur}

1. Byrd JW, Jones KS (2003) Hip arthroscopy in the presence of dysplasia. Arthroscopy 19(10):10551060

2. Byrd JW, Jones KS (2004) Diagnostic accuracy of clinical assessment, magnetic resonance imaging, magnetic resonance arthrography, and intra-articular injection in hip arthroscopy patients. Am J Sports Med 32(7):1668-1674

3. Clohisy JC, Barrett SE, Gordon JE et al (2006) Periacetabular osteotomy in the treatment of severe acetabular dysplasia. Surgical technique. J Bone Joint Surg [Am] 88 [suppl 1 Pt 1]:65-83

4. Clohisy JC, Nunley RM, Carlisle JC et al (2009) Incidence and characteristics of femoral deformities in the dysplastic hip. Clin Orthop Relat Res 467(1):128-134

5. Costa ML, Villar RN (2006) The acetabular labrum. Arthroscopic diagnosis and therapy of degenerate and traumatic lesions. Orthopäde 35(1):54, 56-58

6. Crawford MJ, Dy CJ, Alexander JW et al (2007) The Frank Stinchfield Award. The biomechanics of the hip labrum and the stability of the hip. Clin Orthop Relat Res 465:16-22

7. Cunningham T, Jessel R, Zurakowski D et al (2006) Delayed gadolinium-enhanced magnetic resonance imaging of cartilage to predict early failure of Bernese periacetabular osteotomy for hip dysplasia. J Bone Joint Surg [Am] 88(7):1540-1548

8. Czerny C, Hofmann S, Neuhold A et al (1996) Lesions of the acetabular labrum: accuracy of MR imaging and MR arthrography in detection and staging. Radiology 200(1):225-230

9. Dienst M, Seil R, Kohn DM (2005) Safe arthroscopic access to the central compartment of the hip. Arthroscopy 21(12):1510-1514

10. Espinosa N, Rothenfluh DA, Beck M et al (2006) Treatment of femoro-acetabular impingement: preliminary results of labral refixation. J Bone Joint Surg [Am] 88(5):925-935

11. Ferguson SJ, Bryant JT, Ganz R et al (2000) The acetabular labrum seal: a poroelastic finite element model. Clin Biomech (Bristol, Avon) 15(6):463-468

12. Ferguson SJ, Bryant JT, Ganz R et al (2000) The influence of the acetabular labrum on hip joint cartilage consolidation: a poroelastic finite element model. J Biomech 33(8):953-960

13. Ganz R, Klaue K, Vinh TS et al (1988) A new periacetabular osteotomy for the treatment of hip dysplasias. Technique and preliminary results. Clin Orthop Relat Res (232):26-36

14. Gray AJ, Villar RN (1997) The ligamentum teres of the hip: an arthroscopic classification of its pathology. Arthroscopy 13(5):575-578

15. Gunther KP, Thielemann F, Hartmann A et al (2008) Combined hip-dysplasia and femuroacetabular impingement. Diagnosis and simultaneous surgical treatment. Orthopäde 37(6):577-586

16. Kim YJ, Jaramillo D, Millis MB et al (2003) Assessment of early osteoarthritis in hip dysplasia with delayed gadolinium-enhanced magnetic resonance imaging of cartilage. J Bone Joint Surg [Am] 85A(10):1987-1992

17. Kim YT, Azuma H (1995) The nerve endings of the acetabular labrum. Clin Orthop Relat Res 320:176181

18. Klaue K, Durnin CW, Ganz R (1991) The acetabular rim syndrome. A clinical presentation of dysplasia of the hip. J Bone Joint Surg [Br] 73(3):423-429

19. Leunig M, Beck M, Stauffer E et al (2000) Free nerve endings in the ligamentum capitis femoris. Acta Orthop Scand 71(5):452-454 
20. MacDonald SJ, Garbuz D, Ganz R (1997) Clinical evaluation of the symptomatic young adult hip. Semin Arthroplasty 8:3-9

21. Mast JW, Brunner RL, Zebrack J (2004) Recognizing acetabular version in the radiographic presentation of hip dysplasia. Clin Orthop Relat Res (418):48-53

22. McCarthy JC, Lee JA (2002) Acetabular dysplasia: a paradigm of arthroscopic examination of chondral injuries. Clin Orthop Relat Res (405):122-128

23. Millis MB, Murphy SB, Poss R (1996) Osteotomies about the hip for the prevention and treatment of osteoarthrosis. Instr Course Lect 45:209-226

24. Petersilge CA (2001) MR arthrography for evaluation of the acetabular labrum. Skeletal Radiol 30(8):423-430

25. Philippon MJ (2006) New frontiers in hip arthroscopy: the role of arthroscopic hip labral repair and capsulorrhaphy in the treatment of hip disorders. Instr Course Lect 55:309-316

26. Sarban S, Baba F, Kocabey Y et al (2007) Free nerve endings and morphological features of the ligamentum capitis femoris in developmental dysplasia of the hip. J Pediatr Orthop B 16(5):351-356

27. Seldes RM, Tan V, Hunt J et al (2001) Anatomy, histologic features, and vascularity of the adult acetabular labrum. Clin Orthop Relat Res (382):232-240

28. Siebenrock KA, Leunig M, Ganz R (2001) Periacetabular osteotomy: the Bernese experience. Instr Course Lect 50:239-245

29. Siebenrock KA, Schoeniger R, Ganz R (2003) Anterior femoro-acetabular impingement due to acetabular retroversion. Treatment with periacetabular osteotomy. J Bone Joint Surg [Am] 85A(2):278-286

30. Siebenrock KA, Scholl E, Lottenbach M et al (1999) Bernese periacetabular osteotomy. Clin Orthop Relat Res (363):9-20

31. Steadman JR, Rodkey WG, Rodrigo JJ (2001) Microfracture: surgical technique and rehabilitation to treat chondral defects. Clin Orthop Relat Res 391 [suppl]:362-369

32. Steppacher SD, Tannast M, Ganz R et al (2008) Mean 20-year followup of Bernese periacetabular osteotomy. Clin Orthop Relat Res 466(7):1633-1644

33. Tönnis D (1985) Methodik der Untersuchung. In: Tönnis D (Hrsg) Die operative Behandlung der Hüftdysplasie. Technik und Ergebnisse. Enke, Stuttgart, S 67-85

34. Tönnis D, Behrens K, Tscharani F (1981) A new technique of triple osteotomy for turning dysplastic acetabula in adolescents and adults (author's transl). Z Orthop Ihre Grenzgeb 119(3):253-265

35. Tschauner C, Hofmann S (1998) Labrum lesions in residual dysplasia of the hip joint. Biomechanical considerations on pathogenesis and treatment. Orthopäde 27(11):725-732

36. Tschauner C, Hofmann S, Czerny C (1997) Hip dysplasia. Morphology, biomechanics and therapeutic principles with reference to the acetabular labrum. Orthopäde 26(1):89-108

37. Yasunaga Y, Ikuta Y, Kanazawa T et al (2001) The state of the articular cartilage at the time of surgery as an indication for rotational acetabular osteotomy. J Bone Joint Surg [Br] 83(7):1001-1004

\section{Themenübersicht}

Die Zeitschrift „Arthroskopie“ bietet Ihnen umfassende und aktuelle Beiträge zu interessanten Themenschwerpunkten aus allen Bereichen der Arthroskopie.

Wir haben den Jahrgang 2009 und die Planung für 2010 im Überblick für Sie zusammengestellt:

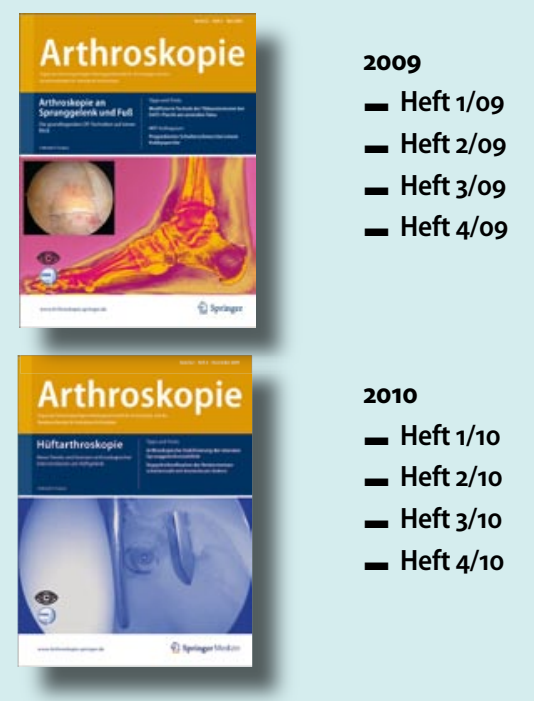

\section{Vorderes und hinteres Kreuzband Arthroskopische Frakturversorgung Patellofemoralgelenk Schultergürtel}

Bestellen Sie einzelne, bereits erschienene Ausgaben oder abonnieren Sie die Zeitschrift zum Preis von EUR 199,- pro Jahr (zzgl. Versandkosten, Ermäßigung für Ärzte in Aus- und Weiterbildung). AGA-Mitglieder erhalten die Zeitschrift im Rahmen Ihrer Mitgliedschaft. Unser Kundenservice steht Ihnen für Fragen und Informationen gerne zur Verfügung:

Springer Customer Service Center GmbH Haberstr. 7, 69126 Heidelberg

Tel.: +49 6221-345-4303, Fax: +49 6221-345-4229

E-Mail: leserservice@springer.com

\section{(1) Beiträge auf einen Blick}

Abonnenten haben online Zugriff auf alle Beiträge im elektronischen Volltextarchiv unter

\section{www.Arthroskopie.springer.de}

Ins Volltextarchiv gelangen Sie von der Startseite über den Navigationspunkt „Online-Archiv".

Nach einer einmaligen Registrierung können Sie die Beiträge als pdf oder in der htmlVersion abrufen.

Eine interessante Lektüre wünscht Ihnen

Ihre Redaktion

Fachzeitschriften Medizin/Psychologie 